\title{
NUTRIX - ARTIFICIAL INTELLIGENCE FOR NUTRITIONISTS
}

\section{INTRODUCTION}

The adherence to a diet is a key to guarantee the desired results in patients who follow a dietary-nutritional treatment. Several authors have found that psychological behavior have a strong influence on diet and have evaluated how personality traits affect eating habits. There are also precedents in the application of artificial intelligence techniques in diets showing that it's attainable to develop tools that help the decision-making process for professionals in the area of dietetics and nutrition.

\section{OBJETIVE}

This work, developed under the NUTRIX Project, has the objective to develop artificial intelligence algorithms based on the open source platform Knime that allows to characterize and predict the adherence of individuals to diet before starting the treatment.

\section{MATERIALS, METHODS \& RESULTS}

108 patients ( 79 women and 29 men between 17 and 51 years old) were characterized anthropometrically and psychologically. The psychological characterization was accomplished applying the Eating Attitudes Test, Eat-40 ; (28,7\% shows an eating disorder, 23 women and 8 men) and the Locus Scale of ROTTER control (33,33\% present an external locus control, 28 women and 8 men). After characterized all the patients were divided into four different types of profiles ("NO_INTERNAL", "YES_INTERNAL", "NO_EXTERNAL", "YES_EXTERNAL" with 65, 7, 12, 24 patients respectively). The anthropometrically characterization includes weight, height, body mass index and lipids percentage. The age of male patients ranges between 17 and 43 years old ( 21 years as the average). BMI ranges between 18,32 and 31,62 and lipids ranges between 8 and 32,7 percent. Female patients have an age range between 17 and 51 years old (20,5 years as the average), an BMI that ranges between 7,13 and 40,64, with lipids that ranges between 12,9 and 45,3 in percentage. Patients were monitored during treatment resulting in $17,6 \%$ (16 women and 3 men) who left the diet before ending the treatment.

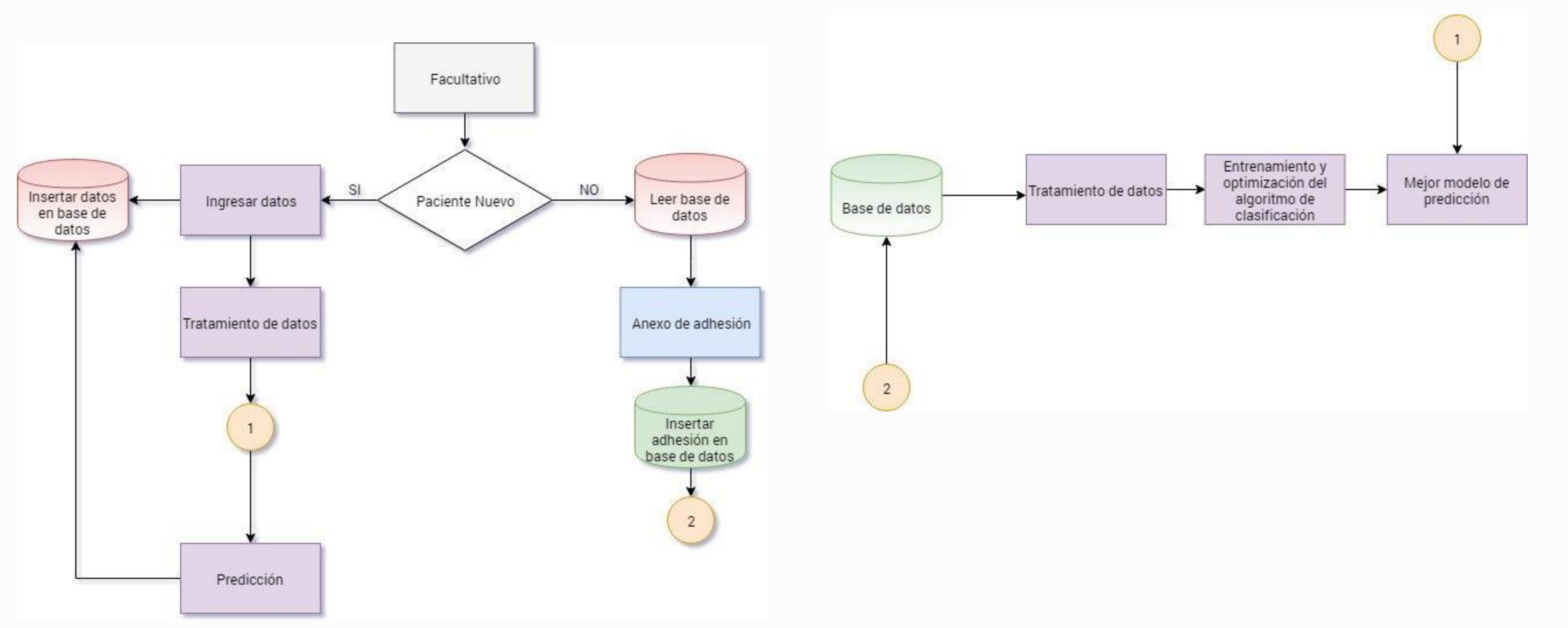

Figure 1. High level Diagram of the Technological Solution

The data was stored in a MySQL database and stratified by adherence (dependent categorical variable) and randomly sampled for training and testing using the $80 \% / 20 \%$ rule. Four machine learning algorithms (random forests, fuzzy logic, neural networks, and self-organized maps) were developed.

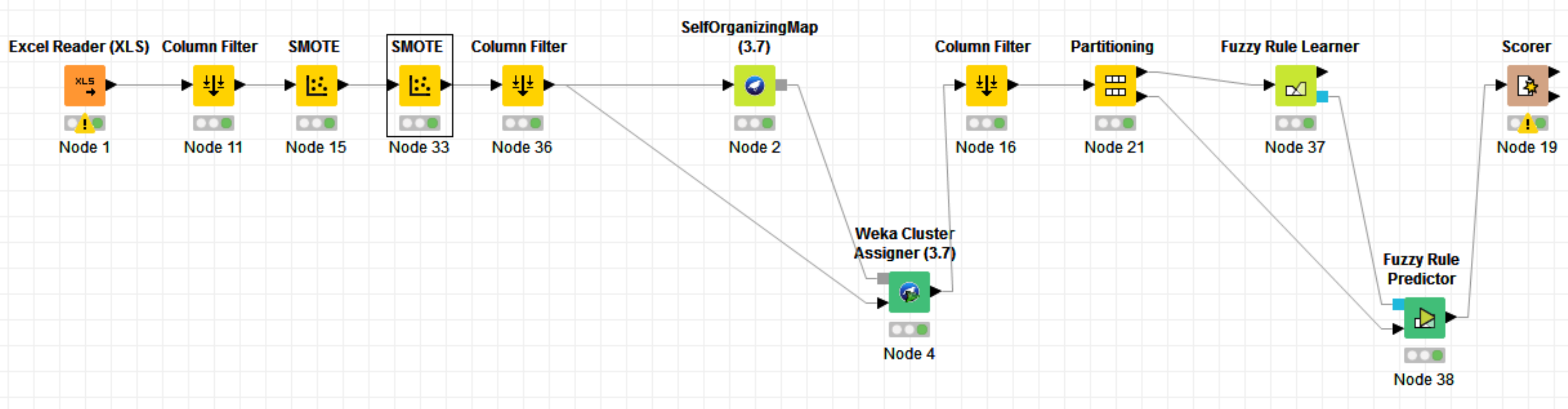

Figure 2. Pre-optimization SOM algorithm

Models were optimized for accuracy by an algorithm that tweaks several parameters in order to provide the most precise results. The optimized algorithms were then validated using crossvalidation and used for prediction. However instead of using the most precise, a scoring system that takes into account the "wisdom" of the four algorithms was developed.

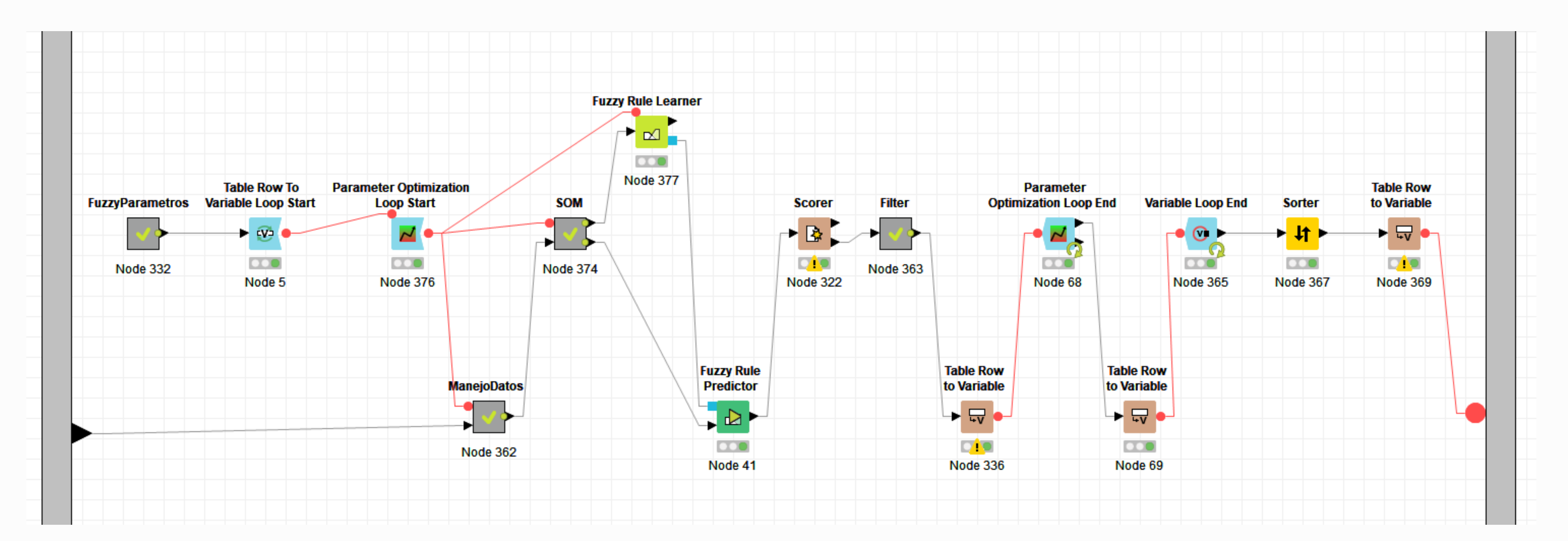

Figure 3. Post-optimization SOM algorithm

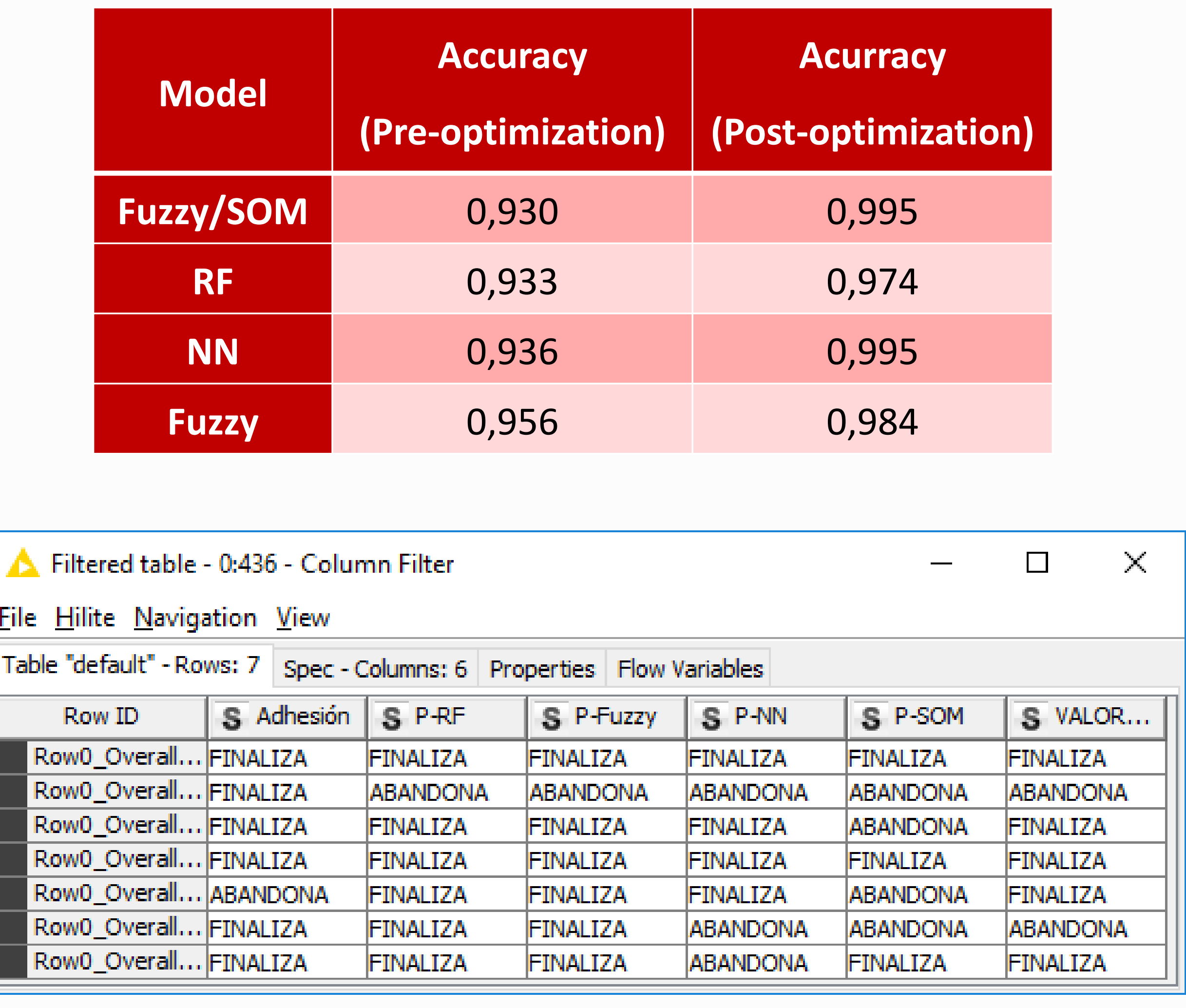

Figure 4. Partial Results of the Scoring System

\section{CONCLUSIONS}

The machine learning algorithms developed under this project have significantly increased the confidence (a priory probability) that a patient leaves the treatment (diet) before starting: from $17,6 \%$ up to $96,5 \%$ which can be used as valuable guidance during the decision-making process of professionals in the area of dietetics and nutrition. 